\title{
ON INFINITESIMAL PROJECTIVE TRANSFORMATIONS OF THE TANGENT BUNDLE WITH THE COMPLETE LIFT OF A FINSLER METRIC
}

\author{
B. Bidabad ${ }^{1}$, M. M. ReZait ${ }^{2}$ and M. Zohrehvand ${ }^{3}$ \\ ${ }^{1}$ Faculty of Mathematics and Computer Science, \\ Amirkabir University of Technology, Tehran, Iran \\ bidabad@aut.ac.ir \\ ${ }^{2}$ Faculty of Mathematics and Computer Science, \\ Amirkabir University of Technology, Tehran, Iran \\ mmreza@aut.ac.ir \\ ${ }^{3}$ Faculty of Mathematical Sciences and Statistics, \\ Malayer University, Malayer, Iran \\ m.zohrehvand@malayeru.ac.ir
}

\begin{abstract}
Let $(M, g)$ be a Finsler manifold and $T M_{0}$ its slit tangent bundle with the complete lift metric $\tilde{g}$. In this paper, we prove that every infinitesimal complete lift projective transformation on $\left(T M_{0}, \tilde{g}\right)$, is an infinitesimal affine transformation. Moreover, if $(M, g)$ is a Landsberg manifold, then there is a one-to-one correspondence between infinitesimal complete lift projective transformations on $\left(T M_{0}, \tilde{g}\right)$ and infinitesimal affine transformations on $(M, g)$.

Key words and Phrases: Finsler manifold, Complete lift metric, Tangent bundle, Infinitesimal projective transformation.
\end{abstract}

\begin{abstract}
Abstrak. Misalkan $(M, g)$ adalah manifold Finsler dan $T M_{0}$ adalah bundel tangen slit-nya dengan metrik lift lengkap $\tilde{g}$. Pada makalah ini, kami membuktikan bahwa setiap transformasi projektif lift lengkap infinitesimal pada $\left(T M_{0}, \tilde{g}\right)$, adalah transformasi afin infinitesimal. Lebih jauh, jika $(M, g)$ adalah manifold Landsberg, maka terdapat korespondensi satu-satu antara transformasi projektif lift lengkap infinitesimal pada $\left(T M_{0}, \tilde{g}\right)$ dengan transformasi afin infinitesimal pada $(M, g)$.

Kata kunci: Manifold Finsler, Metric lift lengkap, Tangent bundle, Infinitesimal projective transformation.
\end{abstract}

2000 Mathematics Subject Classification: 53B40, 53B21, 53C60. Received: 5-Jan-2017, revised: 1-Feb-2017, accepted: 1-Feb-2017. 


\section{INTRODUCTION}

Let $(M, g)$ be a Riemannian manifold and $\phi$ a transformation on $M$. Then, $\phi$ is called a projective transformation if it preserves the geodesics as point sets. Also, an affine transformation may be characterized as a projective transformation which preserves geodesics with the affine parameter.

Let $V$ be a vector field on $M$ and $\left\{\phi_{t}\right\}$ the local one-parameter group generated by $V$. Then, $V$ is called an infinitesimal projective (affine) transformation on $M$ if every $\phi_{t}$ is a projective (affine) transformation.

Let $\tilde{\phi}$ be a transformation of $T M$, the tangent bundle of $M$; then, $\tilde{\phi}$ is called a fiber-preserving transformation if it preserves the fibers. A vector field $\tilde{X}$ on $T M$ with the local one-parameter group $\left\{\tilde{\phi}_{t}\right\}$ is called an infinitesimal fiberpreserving transformation on $T M$ if each $\tilde{\phi}_{t}$ is a fiber-preserving transformation. Infinitesimal fiber-preserving transformations is an important class of infinitesimal transformations on $T M$ which include infinitesimal complete lift transformations as a special subclass(refer to subsection 2.2 or [4]).

It is well-known that there are some lift metrics on $T M$ as follows: complete lift metric or $g_{2}$, diagonal lift metric or Sasaki metric or $g_{1}+g_{3}$, lift metric I+II or $g_{1}+g_{2}$ and lift metic II+III or $g_{2}+g_{3}$, where $g_{1}:=g_{i j} d x^{i} d x^{j}, g_{2}:=2 g_{i j} d x^{i} \delta y^{j}$ and $g_{3}:=g_{i j} \delta y^{i} \delta y^{j}$ are all bilinear differential forms defined globally on $T M$. For more details one can refer to [17].

The problems of exsisting special infinitesimal transformations on the tangent bundle of a Riemannian manifold with some lift metrics are considered by several authors, e.g. $[5,6,7,8,9]$ and $[12,13,14,15]$. The study shows that the special infinitesiaml transformations on $T M$ might lead to some global results. For example, in [7] and [15], it is proved that if $T M$ with the complete lift metric or the lift metric I+II admits an essential infinitesimal conformal ${ }^{1}$ transformation, then $M$ is isomorphic to the standard sphere. Also, it is proved in [12], that if $T M$ with the complete lift metric admits a non-affine infinitesimal projective transformation, then $M$ is locally flat. Therefore, it is meaningful to the study of the infinitesimal transformations on the tangent bundle $T M$.

Yamauchi in [14], proved the following theorem:

Theorem A: Let $M$ be a non-Euclidean complete n-dimensional Riemannian manifold, and let $T M$ be its tangent bundle with the complete lift metric. Then, every infinitesimal fiber-preserving projective transformation on $T M$ is an affine one and it naturally induced an infinitesimal affine transformation on $M$.

Special infinitesimal transformations on the tangent bundle of a Finsler manifold are considered by many authors, e.g. $[1,2,4,10]$.

\footnotetext{
${ }^{1} \mathrm{~A}$ vector field $\tilde{X}$ on $(T M, \tilde{g})$ is called an essential infinitesimal conformal transformation if there exsits a scalar function $\Omega$ on $T M$ such that $\Omega$ depends only $y^{i}$ with respect to the induced coordinate $\left(x^{i}, y^{i}\right)$ on $T M$ and $£_{\tilde{X}} \tilde{g}=2 \Omega \tilde{g},[16]$.
} 
In this paper, the infinitesimal fiber-preserving projective transformations on the tangent bundle of a Finsler manifold with the complete lift metric are considered. Then, as a special case, the infinitesimal complete lift projective transformations are studied and the following theorem is proved.

Theorem 1.1. Let $(M, g)$ be a $C^{\infty}$ connected Finsler manifold and $T M_{0}$ its slit tangent bundle with the complete lift metric $\tilde{g}$. Then, every infinitesimal complete lift projective transformation on $\left(T M_{0}, \tilde{g}\right)$ is affine and it naturally induced an infinitesimal affine transformation on $(M, g)$.

Thus, Theorem A is true for the Finsler manifold and the infinitesimal complete lift transformations. From Theorem 1.1, the following corollary can be immediately found.

Corollary 1.2. The Lie algebra of complete lift projective vector field on $\left(T M_{0}, \tilde{g}\right)$ is reduced to an affine one.

The Landsberg manifolds form an important class of the Finsler manifolds which include the Berwald manifolds. In the next theorem, it is shown that the inverse of Theorem 1.1 is true for the Landsberg manifolds.

Theorem 1.3. Let $(M, g)$ be an n-dimensional Landsberg manifold and $T M_{0}$ its slit tangent bundle with a complete lift metric $\tilde{g}$. Then, every infinitesimal complete lift transformation $V^{c}$ on $\left(T M_{0}, \tilde{g}\right)$ is projective if and only if $V$ is an infinitesimal affine transformation on $(M, g)$.

\section{Preliminaries}

Let $M$ be a real n-dimensional $C^{\infty}$ manifold and $T M$ its tangent bundle. The elements of $T M$ are denoted by $(x, y)$ with $y \in T_{x} M$. Also $T M_{0}=T M \backslash\{0\}$ be the slit tangent bundle of $M$. The natural projection $\pi: T M_{0} \rightarrow M$ is given by $\pi(x, y):=x$. A Finsler structure on $M$ is a function of $F: T M \rightarrow[0, \infty)$ with the following properties; (i) $F$ is $C^{\infty}$ on $T M_{0}$, (ii) $F$ is positively 1-homogeneous on the fibers of tangent bundle $T M$ and (iii) the Hessian $g$ of $F^{2}$ with elements $g_{i j}(x, y):=\frac{1}{2}\left[F^{2}(x, y)\right]_{y^{i} y^{j}}$ is positive-definite. In the sequel of this paper, a Finsler manifold with a Finsler structure $F$ will be denoted by $(M, g)$ instead of $(M, F)$.

Let $V_{v} T M:=k e r \pi_{*}^{v}$ be the set of the vectors tangent to the fiber through $v \in$ $T M_{0}$. Then, the vertical vector bundle on $M$ is defined by $V T M:=\bigcup_{v \in T M_{0}} V_{v} T M$. A non-linear connection or a horizontal distribution on $T M_{0}$ is a complementary distribution $H T M$ for $V T M$ on $T T M_{0}$. Therefore, by using a non-linear connection, the following decomposition is resulted:

$$
T T M_{0}=V T M \oplus H T M,
$$

where HTM is a vector bundle completely determined by the non-linear differentiable functions $N^{i}{ }_{j}(x, y)$ on $T M$, which is called coefficients of the non-linear 
connection $H T M$. The pair $(H T M, \nabla)$ is called a Finsler connection on the manifold $M$, where $\nabla$ a linear connection to $V T M$. Indeed, a Finsler connection is a triple $\left(N^{i}{ }_{j}, F_{j k}^{i}, C_{j k}^{i}\right)$ where $N^{i}{ }_{j}$ are the coefficients of a nonlinear connection, $F^{i}{ }_{j k}$ and $C^{i}{ }_{j k}$ are the horizontal part and the vertical part of this connection, respectively[3].

Using the local coordinates $\left(x^{i}, y^{i}\right)$ on $T M$ we have the local field of frames field $\left\{X_{i}, X_{\bar{i}}\right\}$ on $T T M$. It is well known that a local field of frames $\left\{X_{i}, X_{\bar{i}}\right\}$ can be chosen so that it is adapted to the decomposition (1) i.e. $X_{i} \in \Gamma(H T M)$ and $X_{\bar{i}} \in \Gamma(V T M)$ set of vector fields on $H T M$ and $V T M$, where

$$
X_{i}:=\frac{\partial}{\partial x^{i}}-N^{j}{ }_{i} \frac{\partial}{\partial y^{j}}, X_{\bar{i}}:=\frac{\partial}{\partial y^{i}},
$$

where the indices $i, j, \ldots$ and $\bar{i}, \bar{j}, \ldots$ run over the range $1, \ldots, n$.

Analogous to Riemannian geometry, the following lemma in Finsler geometry was obtained by straightforward calculations.

Lemma 2.1. [14] The Lie brackets of the adapted frame of TM satisfy the following

(1) $\left[X_{i}, X_{j}\right]=R^{h}{ }_{i j} X_{\bar{h}}$,

(2) $\left[X_{i}, X_{\bar{j}}\right]=X_{\bar{j}}\left(N_{i}^{h}\right) X_{\bar{h}}$,

(3) $\left[X_{\bar{i}}, X_{\bar{j}}\right]=0$,

where $R_{i j}^{h}=X_{j}\left(N_{i}^{h}\right)-X_{i}\left(N_{j}^{h}\right)$.

For a Finsler connection $\left(N_{j}^{i}, F_{j k}^{i}, C_{j k}^{i}\right)$, the curvature tensor has three component $R, P$ and $S$, that are called hh-curvature, hv-curvature and vv-curvature, respectively. They are defined as follows:

$$
\begin{gathered}
R_{k i j}^{h}:=X_{i}\left(F_{k j}^{h}\right)-X_{j}\left(F_{k i}^{h}\right)+F_{k j}^{m} F_{m i}^{h}-F_{k i}^{m} F_{m j}^{h}+R_{i j}^{m} C_{j m}^{h}, \\
P_{k i j}^{h}:=X_{\bar{k}}\left(F_{i j}^{h}\right)-X_{j}\left(C_{i k}^{h}\right)+F_{k i}^{m} C_{m j}^{h}-C_{k j}^{m} F_{m i}^{h}+X_{\bar{j}}\left(N_{i}^{m}\right) C_{j m}^{h}, \\
S_{k i j}^{h}:=X_{\bar{j}}\left(C_{k i}^{h}\right)-X_{\bar{i}}\left(C_{k j}^{h}\right)+C_{k i}^{m} C_{m j}^{h}-C_{k j}^{m} C_{m i}^{h} .
\end{gathered}
$$

Let $(M, g)$ be a Finsler manifold, the geodesic of $g$ satisfy the following system of differential equations

$$
\frac{d^{2} x^{i}}{d t^{2}}+2 G^{i}\left(x, \frac{d x}{d t}\right)=0,
$$

where $G^{i}=G^{i}(x, y)$ are called the geodesic coefficients, which are given by

$$
G^{i}=\frac{1}{4} g^{i l}\left\{\left[F^{2}\right]_{x^{m} y^{l}} y^{m}-\left[F^{2}\right]_{x^{l}}\right\} .
$$

The differentiable functions $G^{i}{ }_{j}:=X_{\bar{j}}\left(G^{i}\right)$ determine a non-linear connection which is called the canonical nonlinear connection of Finsler manifold $(M, g)$. In what follows, the canonical nonlinear connection $G^{i}{ }_{j}$ will be used.

There are several Finsler connections on a Finsler manifold, which we present some of them. The Berwald connection of a Finsler manifold $(M, g)$ is defined by 
triple $\left(G^{i}{ }_{j}, G_{j k}^{i}, 0\right)$, where $G_{j k}^{i}:=X_{\bar{k}}\left(G_{j}^{i}\right)$. The $h h$-curvature $H_{k}{ }^{h}{ }_{i j}$ and $h v$ curvature $G_{k}{ }_{i j}$ of the Berwald connection are obtained as follows:

$$
\begin{gathered}
H_{k i j}{ }_{i j}=X_{i}\left(G_{k j}^{h}\right)-X_{j}\left(G_{k i}^{h}\right)+G_{k j}^{m} G_{m i}^{h}-G_{k i}^{m} G_{m j}^{h}, \\
G_{k i j}^{h}=X_{\bar{k}}\left(G^{h}{ }_{i j}\right),
\end{gathered}
$$

respectively. It is obvious that $R_{i j}^{h}=y^{k} H_{k i j}{ }_{i j}$.

Also, the Cartan connection of a Finsler manifold $(M, g)$ is defined by triple $\left(G_{j}^{i}, F_{j k}^{i}, C_{j k}^{i}\right)$, where $F_{j k}^{i}:=\frac{1}{2} g^{i h}\left\{X_{j}\left(g_{k h}\right)+X_{k}\left(g_{j h}\right)-X_{h}\left(g_{j k}\right)\right\}$ and $C^{i}{ }_{j k}:=$ $\frac{1}{2} g^{i h} X_{\bar{k}}\left(g_{j h}\right)$.

A Finsler manifold $(M, g)$ is called a Berwald manifold, if the geodesic spray coefficient $G^{i}$ s are quadratic functions of $y$-coordinates in each tangent space, i.e.

$$
X_{\bar{l}} X_{\bar{k}} X_{\bar{j}}\left(G^{i}\right)=0, \quad \forall j, k, l .
$$

In the other words, the Finsler manifold $(M, g)$ is a Berwald metric if the hvcurvature tensor field of the Berwald connection vanishes.

A Finsler manifold $(M, g)$ is called Landsberg manifold, if the geodesic spary coefficient $G^{i}$ 's satisfy the following equations [11]

$$
y^{m} g_{i m} X_{\bar{l}} X_{\bar{k}} X_{\bar{j}}\left(G^{i}\right)=0, \quad \forall j, k, l .
$$

It is obvious that every Brwald manifold is a Landsberg manifold.

By defining the tensor field $P^{i}{ }_{j k}:=G^{i}{ }_{j k}-F^{i}{ }_{j k}$, one can see that, a Finsler manifold $(M, g)$ is a Landsberg manifold if and only if $P_{j k}^{i}=0$. For more details, one can refer to [3]. In the following, all manifolds are supposed to be connected.

2.1. Infinitesimal fiber-preserving transformations. Let $\tilde{X}$ be a vector field on $T M$ and $\left\{\tilde{\phi}_{t}\right\}$ the local one-parameter group of local transformations of $T M$ generated by $\tilde{X}$. Then, $\tilde{X}$ is called a fiber-preserving vector field on $T M$ if each $\tilde{\phi}_{t}$ is a fiber-preserving transformation of $T M$. From [13], we have the following lemma.

Lemma 2.2. Let $\tilde{X}$ be a vector field on $T M$ with components $\left(v^{h}, v^{\bar{h}}\right)$ with respect to the adapted frame $\left\{X_{h}, X_{\bar{h}}\right\}$. Then $\tilde{X}$ is a fiber-preserving vector field on TM if and only if $v^{h}$ are functions on $M$

Therefore, every fiber-preserving vector field $\tilde{X}$ on $T M$ induces a vector field $V=v^{h} \partial_{h}$ on $M$, where $\partial_{i}:=\frac{\partial}{\partial x^{i}}$.

Let $\left\{d x^{i}, \delta y^{i}\right\}$ be the dual basis of $\left\{X_{h}, X_{\bar{h}}\right\}$, where $\delta y^{i}=d y^{i}-G^{i}{ }_{h} d x^{h}$. Using a straight forward calculation similar to [14], one can obtain the following lemma.

Lemma 2.3. Let $\tilde{X}$ be a fiber-preserving vector field of $T M$ with the components $\left(v^{h}, v^{\bar{h}}\right)$. Then, the Lie derivatives of the adapted frame and the dual basis are given as follows:

(1) $£_{\tilde{X}} X_{h}=-\partial_{h} v^{m} X_{m}+\left\{v^{b} R_{b h}^{m}-v^{\bar{b}} G_{b h}^{m}-X_{h}\left(v^{\bar{h}}\right)\right\} X_{\bar{m}}$,

(2) $£_{\tilde{X}} X_{\bar{h}}=\left\{v^{b} G_{b h}^{m}-X_{\bar{h}}\left(v^{\bar{m}}\right)\right\} X_{\bar{m}}$, 
(3) $£_{\tilde{X}} d x^{h}=\partial_{m} v^{h} d x^{m}$,

(4) $£_{\tilde{X}} \delta y^{h}=-\left\{v^{b} R_{b m}^{h}-v^{\bar{b}} G_{b m}^{h}-X_{m}\left(v^{\bar{h}}\right)\right\} d x^{m}-\left\{v^{b} G_{b m}^{h}-X_{\bar{m}}\left(v^{\bar{h}}\right)\right\} \delta y^{m}$.

For more details in infinitesimal transformations, one can refer to [8].

2.2. Complete Lift Vector Fields and Lie Derivative. Let $V=v^{i} \partial_{i}$ be a vector field on $M$. Then, $V$ induces an infinitesimal point transformation on $M$. This is naturally extended to a point transformation of the tangent bundle $T M$ which is called extended point transformation. If $\left\{\varphi_{t}\right\}$ is the local 1-parameter group of $M$ generated by $V$ and $\tilde{\varphi}_{t}$ the extended point transformation of $\varphi_{t}$, then $\left\{\tilde{\varphi}_{t}\right\}$ induces a vector field $V^{c}$ on $T M$ which is called the complete lift of $V$, (c.f. $[4])$.

The complete lift vector field $V^{c}$ of $V$ can be written as $V^{c}=v^{i} X_{i}+$ $y^{j}\left(F_{j a}^{i} v^{a}+\partial_{j} v^{i}\right) X_{\bar{i}}$. From the Lemma 2.2, it concluded that, the class of complete lift vector fields is a subclass of fiber-preserving vector fields.

Let $V$ be a vector field on $M$ and $\left\{\varphi_{t}\right\}$ the local one parameter group of local transformations of $M$ generated by $V$. Take any tensor field $S$ on $M$. Then, the Lie derivative $£_{V} S$ of $S$ with respect to $V$ is a tensor field on $M$, defined by

$$
£_{V} S=\left.\frac{\partial}{\partial t} \varphi_{t}^{*}(S)\right|_{t=0}=\lim _{t \rightarrow 0} \frac{\varphi_{t}^{*}(S)-S}{t},
$$

on the domain of $\varphi_{t}$, where $\varphi_{t}^{*}(S)$ denotes the pull back of $S$ by $\varphi_{t}$.

In local coordinates the Lie derivative of an arbitrary tensor, $T_{i}^{k}$, is given locally by[16];

$$
£_{V} T_{i}^{k}=v^{a} \partial_{a} T_{i}^{k}+y^{a} \partial_{a} v^{b} X_{\bar{b}}\left(T_{i}^{k}\right)-T_{i}^{a} \partial_{a} v^{k}+T_{a}^{k} \partial_{i} v^{a} .
$$

Therefore

$$
£_{V} y^{i}=0 .
$$

For the Lie derivatives of the adapted frame and the dual basis with respect to complete lift vector field $V^{c}$, the following lemma can be presented.

Lemma 2.4. [4] Let $(M, g)$ be a Finsler manifold, $V$ a vector field on $M$ and $V^{c}$ its complete lift, then

(1) $£_{V^{c}} X_{i}=-\partial_{i} v^{h} X_{h}-£_{V} G_{i}^{h} X_{\bar{h}}$,

(2) $£_{V^{c}} X_{\bar{i}}=-\partial_{i} v^{h} X_{\bar{h}}$,

(3) $£_{V^{c}} d x^{h}=\partial_{m} v^{h} d x^{m}$,

(4) $£_{V^{c}} \delta y^{h}=£_{V} G_{m}^{h} d x^{m}+\partial_{m} v^{h} \delta y^{m}$.

2.3. Infinitesimal projective transformations. Let $M$ be a Riemannian manifold. A vector field $X$ on $M$ is said to be an infinitesimal projective transformation, if there exists a 1-form $\theta$ on $M$ such that

$$
\left(£_{X} \nabla\right)(Y, Z)=\theta(Y) Z+\theta(Z) Y,
$$

or equivalently

$$
£_{X}\left(\nabla_{Y} Z\right)-\nabla_{Y}\left(£_{X} Z\right)-\nabla_{[X, Y]} Z=\theta(Y) Z+\theta(Z) Y .
$$


where $\nabla$ is the Riemannian connection of $M$ and $Y, Z \in \Gamma(M)$ the set of vector fields on $M$.

In Finsler geometry, a vector field $V$ on $(M, g)$ is called an infinitesimal projective transformation if there exists a function $\Psi(x, y)$ on $T M$ such that $£_{V} G^{i}=\Psi y^{i}$ [2]. Accordingly, the following relations can be resulted.

$$
\begin{gathered}
£_{V} G_{j}^{i}=\Psi \delta_{j}^{i}+\Psi_{j} y^{i}, \\
£_{V} G^{i}{ }_{j k}=\Psi_{k} \delta_{j}^{i}+\Psi_{j} \delta_{k}^{i}+\Psi_{j k} y^{i}, \\
£_{V} G^{i}{ }_{j k l}=\Psi_{k l} \delta_{j}^{i}+\Psi_{j l} \delta_{k}^{i}+\Psi_{j k} \delta_{l}^{i}+\Psi_{j k l} y^{i},
\end{gathered}
$$

where $\Psi_{j}:=X_{\bar{j}}(\Psi), \Psi_{j k}:=X_{\bar{k}}\left(\Psi_{j}\right)$ and $\Psi_{j k l}:=X_{\bar{l}}\left(\Psi_{j k}\right)$. If $\Psi=0$, then it can be said that $V$ is an infinitesimal affine transformation.

\section{Riemannian Connection of $T M_{0}$ With the Complete Lift Metric}

Let $g=\left(g_{i j}(x, y)\right)$ be a Finsler metric on $M$. As we said that, there are several Riemannian or pseudo-Riemannian metrics on $T M_{0}$ which can be defined from $g$. They are called the lift metric of $g$. A one of such metrics is $\tilde{g}=2 g_{i j} d x^{i} \delta y^{j}$, which is called the complete lift metric, (c.f. [17]). Thus $\left(T M_{0}, \tilde{g}\right)$ is a Riemannian manifold.

Let $\tilde{\nabla}$ be the Riemannian connection of $T M_{0}$ with respect to the complete lift metric $\tilde{g}$ and $\tilde{\Gamma}_{B C}^{A}$ the coefficients of $\tilde{\nabla}$, that is,

$$
\begin{aligned}
& \tilde{\nabla}_{X_{i}} X_{j}=\tilde{\Gamma}_{j i}^{m} X_{m}+\tilde{\Gamma}_{j i}^{\bar{m}} X_{\bar{m}}, \quad \tilde{\nabla}_{X_{i}} X_{\bar{j}}=\tilde{\Gamma}_{\bar{j} i}^{m} X_{m}+\tilde{\Gamma}_{\bar{j} i}^{\bar{m}} X_{\bar{m}}, \\
& \tilde{\nabla}_{X_{\bar{i}}} X_{j}=\tilde{\Gamma}_{j \bar{i}}^{m} X_{m}+\tilde{\Gamma}_{j \bar{i}}^{\bar{m}} X_{\bar{m}}, \quad \tilde{\nabla}_{X_{\bar{i}}} X_{\bar{j}}=\tilde{\Gamma}_{\bar{j} \bar{i}}^{m} X_{m}+\tilde{\Gamma}_{\bar{j} \bar{i}}^{\bar{m}} X_{\bar{m}},
\end{aligned}
$$

where the coefficients $A, B, C, \ldots$ run over the range $1, \ldots, n, \overline{1}, \ldots, \bar{n}$.

The following lemma is trivial.

Lemma 3.1. We have the following equations

(1) $\tilde{\nabla}_{X_{i}} d x^{h}=-\tilde{\Gamma}_{m i}^{h} d x^{m}-\tilde{\Gamma}_{\bar{m} i}^{h} \delta y^{m}$,

(2) $\tilde{\nabla}_{X_{i}} \delta y^{h}=-\tilde{\Gamma}_{m i}^{\bar{h}} d x^{m}-\tilde{\Gamma}_{\bar{m} i}^{\bar{h}} \delta y^{m}$,

(3) $\tilde{\nabla}_{X_{\bar{i}}} d x^{h}=-\tilde{\Gamma}_{m \bar{i}}^{h} d x^{m}-\tilde{\Gamma}_{\bar{m} \bar{i}}^{h} \delta y^{m}$,

(4) $\tilde{\nabla}_{X_{\bar{i}}} \delta y^{h}=-\tilde{\Gamma}_{m \bar{i}}^{\bar{h}} d x^{m}-\tilde{\Gamma}_{\bar{m} \bar{i}}^{\bar{h}} \delta y^{m}$.

Since the torsion tensor $T(X, Y)$ of $\tilde{\nabla}$ defined by $T(X, Y)=\tilde{\nabla}_{X} Y-\tilde{\nabla}_{Y} X-$ $[X, Y]$ vanishes, the following relations can be given by means of Lemma 2.1 and the relation $(3)$

$$
\begin{gathered}
\tilde{\Gamma}_{j i}^{m}=\tilde{\Gamma}_{i j}^{m}, \quad \tilde{\Gamma}_{j i}^{\bar{m}}=\tilde{\Gamma}_{i j}^{\bar{m}}+R_{i j}^{m}, \\
\tilde{\Gamma}_{\bar{j} i}^{m}=\tilde{\Gamma}_{i \bar{j}}^{m}, \quad \tilde{\Gamma}_{\bar{j} i}^{\bar{m}}=\tilde{\Gamma}_{i \bar{j}}^{\bar{m}}+G_{j i}^{m}, \\
\tilde{\Gamma}_{\bar{j} \bar{i}}^{m}=\tilde{\Gamma}_{\bar{i} \bar{j}}^{m}, \quad \tilde{\Gamma}_{\bar{j} i}^{\bar{m}}=\tilde{\Gamma}_{\bar{i} \bar{j}}^{\bar{m}} .
\end{gathered}
$$


Lemma 3.2. The connection coefficients $\tilde{\Gamma}_{B C}^{A}$ of $\tilde{\nabla}$ satisfy the following relations.
a) $\tilde{\Gamma}_{j i}^{h}=F_{j i}^{h}-P_{j i}^{h}$
b) $\tilde{\Gamma}_{j i}^{\bar{h}}=g^{h m} R_{i m j}$,
c) $\tilde{\Gamma}_{j i}^{h}=0$,
d) $\tilde{\Gamma}_{j \bar{i}}^{h}=0$,
e) $\tilde{\Gamma}_{\bar{j} i}^{\bar{h}}=G^{h}{ }_{j i}$,
f) $\tilde{\Gamma}_{j \bar{i}}^{\bar{h}}=0$,
g) $\tilde{\Gamma}_{\overline{j i}}^{h}=0$,
h) $\tilde{\Gamma}_{\overline{j i}}^{\bar{h}}=2 C_{j i}^{h}$.

Proof. By means of Lemma 3.1 and metric compatibility of $\tilde{\nabla}$, i.e., $\tilde{\nabla} \tilde{g}=0$, we have

$0=\tilde{\nabla}_{X_{m}} \tilde{g}=\tilde{\nabla}_{X_{m}}\left(2 g_{i j} d x^{i} \delta y^{j}\right)$

$=-2 g_{i a} \tilde{\Gamma}_{j m}^{\bar{a}} d x^{i} d x^{j}+2\left\{g_{a j}\left(F_{i m}^{a}-\tilde{\Gamma}_{i m}^{a}\right)+g_{i a}\left(F_{j m}^{a}-\tilde{\Gamma}_{\bar{j} m}^{\bar{a}}\right)\right\} d x^{i} \delta y^{j}-2 g_{a j} \tilde{\Gamma}_{i m}^{a} \delta y^{i} \delta y^{j}$. and

$$
\begin{aligned}
0 & =\tilde{\nabla}_{X_{\bar{m}}} \tilde{g}=\tilde{\nabla}_{X_{\bar{m}}}\left(2 g_{i j} d x^{i} \delta y^{j}\right) \\
& =-2 g_{i a} \tilde{\Gamma}_{j \bar{m}}^{\bar{a}} d x^{i} d x^{j}+2\left\{2 C_{i j m}-g_{a j} \tilde{\Gamma}_{i \bar{m}}^{a}-g_{i a} \tilde{\Gamma}_{\bar{j} \bar{m}}^{\bar{a}}\right\} d x^{i} \delta y^{j}-2 g_{a j} \tilde{\Gamma}_{\bar{i} \bar{m}}^{a} \delta y^{i} \delta y^{j} .
\end{aligned}
$$

It follows that

$$
\begin{gathered}
g_{i a} \tilde{\Gamma}_{j m}^{\bar{a}}+g_{j a} \tilde{\Gamma}_{i m}^{\bar{a}}=0, \\
g_{a j}\left(F_{i m}^{a}-\tilde{\Gamma}_{i m}^{a}\right)+g_{i a}\left(F_{j m}^{a}-\tilde{\Gamma}_{\bar{j} m}^{\bar{a}}\right)=0, \\
g_{a j} \tilde{\Gamma}_{\bar{i} m}^{a}+g_{a i} \tilde{\Gamma}_{\bar{j} m}^{a}=0, \\
g_{i a} \tilde{\Gamma}_{j \bar{m}}^{\bar{a}}+g_{j a} \tilde{\Gamma}_{i \bar{m}}^{\bar{a}}=0, \\
2 C_{i j m}-g_{a j} \tilde{\Gamma}_{i \bar{m}}^{a}-g_{i a} \tilde{\Gamma}_{\bar{j} \bar{m}}^{\bar{a}}=0, \\
g_{a j} \tilde{\Gamma}_{\bar{i} \bar{m}}^{a}+g_{a i} \tilde{\Gamma}_{\bar{j} \bar{m}}^{a}=0 .
\end{gathered}
$$

From (4) and (5), we have

$$
\begin{aligned}
g_{i a} \tilde{\Gamma}_{j m}^{\bar{a}} & =-g_{j a} \tilde{\Gamma}_{i m}^{\bar{a}}=-g_{j a}\left(\tilde{\Gamma}_{m i}^{\bar{a}}+R_{m i}^{a}\right)=-R_{j m i}-g_{j a} \tilde{\Gamma}_{m i}^{\bar{a}} \\
& =-R_{j m i}+g_{m a}\left(\tilde{\Gamma}_{i j}^{\bar{a}}+R_{i j}^{a}\right)=-R_{j m i}+R_{m i j}+g_{m a} \tilde{\Gamma}_{i j}^{\bar{a}} \\
& =-R_{j m i}+R_{m i j}-g_{i a} \tilde{\Gamma}_{m j}^{\bar{a}}=-R_{j m i}+R_{m i j}-g_{i a}\left(\tilde{\Gamma}_{j m}^{\bar{a}}+R_{j m}^{a}\right) \\
& =-R_{j m i}+R_{m i j}-R_{i j m}-g_{i a} \tilde{\Gamma}_{j m}^{\bar{a}}=-g_{i a} \tilde{\Gamma}_{j m}^{\bar{a}}+2 R_{m i j},
\end{aligned}
$$

which show the relation $b$ ) in the lemma.

According to (4) and (10):

$$
g_{a j} \tilde{\Gamma}_{\bar{i} \bar{m}}^{a}=-g_{a i} \tilde{\Gamma}_{\bar{j} \bar{m}}^{a}=-g_{a i} \tilde{\Gamma}_{\bar{m} \bar{j}}^{a}=g_{a m} \tilde{\Gamma}_{\bar{i} \bar{j}}^{a}=g_{a m} \tilde{\Gamma}_{\bar{j} \bar{i}}^{a}=-g_{a j} \tilde{\Gamma}_{\bar{m} \bar{i}}^{a}=-g_{a j} \tilde{\Gamma}_{\bar{i} \bar{m}}^{a},
$$

thus, we get the relation $g$ ) in the lemma.

From (4) and (6), we have

$$
\begin{aligned}
g_{a j}\left(F_{i m}^{a}-\tilde{\Gamma}_{i m}^{a}\right) & =-g_{i a}\left(F_{j m}^{a}-\tilde{\Gamma}_{\bar{j} m}^{\bar{a}}\right)=-g_{i a}\left(G_{j m}^{a}-P_{j m}^{a}-\tilde{\Gamma}_{\bar{j} m}^{\bar{a}}\right) \\
& =-g_{i a}\left(-P_{j m}^{a}-\tilde{\Gamma}_{m \bar{j}}^{\bar{a}}\right) .
\end{aligned}
$$

From above and (8), it can be said that

$$
g_{a j}\left(F_{i m}^{a}-\tilde{\Gamma}_{i m}^{a}\right)+g_{a j}\left(F_{m i}^{a}-\tilde{\Gamma}_{m i}^{a}\right)=g_{i a} P_{j m}^{a}+g_{m a} P_{j i}^{a}=2 P_{j i m},
$$

this shows the relations $a$ ), $e$ ) and $f$ ). 
From (7) and (9), it can be said that

$$
g_{a i} \tilde{\Gamma}_{\bar{j} m}^{a}=-g_{a j} \tilde{\Gamma}_{\bar{i} m}^{a}=-g_{a j} \tilde{\Gamma}_{m \bar{i}}^{a}=g_{m a} \tilde{\Gamma}_{\bar{j} \bar{i}}^{\bar{a}}-2 C_{m j i} .
$$

From (4), (9) and (11), the relations $c$ ), $d$ ) and $h$ ) can be obtained. This completes the proof.

Remark 3.1. From Lemma 3.2 and the relation (3), the following equations can be obtained.

$$
\begin{aligned}
\tilde{\nabla}_{X_{i}} X_{j}= & \left(F_{j i}^{h}-P_{j i}^{h}\right) X_{h}+g^{h m} R_{i m j} X_{\bar{h}}, \quad \tilde{\nabla}_{X_{i}} X_{\bar{j}}=G_{j i}^{h} X_{\bar{h}}, \\
& \tilde{\nabla}_{X_{\bar{i}}} X_{j}=0, \quad \tilde{\nabla}_{X_{\bar{i}}} X_{\bar{j}}=2 C_{j i}^{h} X_{\bar{h}} .
\end{aligned}
$$

It would be mentioned that, when $(M, g)$ is a Riemannian manifold, then the relations (12) are reduced to

$$
\begin{aligned}
\tilde{\nabla}_{X_{i}} X_{j}= & \Gamma_{j i}^{h} X_{h}+g^{h m} R_{i m j} X_{\bar{h}}, \quad \tilde{\nabla}_{X_{i}} X_{\bar{j}}=\Gamma_{j i}^{h} X_{\bar{h}}, \\
& \tilde{\nabla}_{X_{\bar{i}}} X_{j}=0, \quad \tilde{\nabla}_{X_{\bar{i}}} X_{\bar{j}}=0,
\end{aligned}
$$

where $\Gamma_{j i}^{h}$ are the coefficients of the Riemannian connection of $M$.

\section{Main Results}

Let $(M, g)$ be a Finsler manifold and $T M_{0}$ its slit tangent bundle with complete lift metric $\tilde{g}$. Here, infinitesimal fiber-preserving projective transformations on $\left(T M_{0}, \tilde{g}\right)$ are considered and the following proposition is proved.

Proposition 4.1. Let $(M, g)$ be a n-dimensional Finsler manifold and $T M_{0}$ its slit tangent bundle with the complete lift metric $\tilde{g}$. Then, every infinitesimal fiberpreserving projective transformation on $\left(T M_{0}, \tilde{g}\right)$ induces an infinitesimal projective transformation on $(M, g)$.

Proof. Let $\tilde{X}$ be an infinitesimal fiber-preserving projective transformation on $T M_{0}$. From (2), it can be concluded that there exists a 1 -form $\Theta$ on $T M_{0}$ such that

$$
£_{\tilde{X}} \tilde{\nabla}_{\tilde{Y}} \tilde{Z}-\tilde{\nabla}_{\tilde{Y}} £_{\tilde{X}} \tilde{Z}-\tilde{\nabla}_{[\tilde{X}, \tilde{Y}]} \tilde{Z}=\Theta(\tilde{Y}) \tilde{Z}+\Theta(\tilde{Z}) \tilde{Y}
$$

where $\tilde{Y}$ and $\tilde{Z} \in \Gamma(T M)$. Let $\tilde{X}=v^{h} X_{h}+v^{\bar{h}} X_{\bar{h}}$ and $\Theta=\theta_{i} d x^{i}+\theta_{\bar{i}} \delta y^{i}$. We obtain the following.

$$
\begin{aligned}
& £_{\tilde{X}} \tilde{\nabla}_{X_{\bar{i}}} X_{j}-\tilde{\nabla}_{X_{\bar{i}}} £_{\tilde{X}} X_{j}-\tilde{\nabla}_{\left[\tilde{X}, X_{\bar{i}}\right]} X_{j}=\Theta\left(X_{\bar{i}}\right) X_{j}+\Theta\left(X_{j}\right) X_{\bar{i}}, \\
& £_{\tilde{X}} \tilde{\nabla}_{X_{\bar{i}}} X_{\bar{j}}-\tilde{\nabla}_{X_{\bar{i}}} £_{\tilde{X}} X_{\bar{j}}-\tilde{\nabla}_{\left[\tilde{X}, X_{\bar{i}}\right]} X_{\bar{j}}=\Theta\left(X_{\bar{i}}\right) X_{\bar{j}}+\Theta\left(X_{\bar{j}}\right) X_{\bar{i}}, \\
& £_{\tilde{X}} \tilde{\nabla}_{X_{i}} X_{j}-\tilde{\nabla}_{X_{i}} £_{\tilde{X}} X_{j}-\tilde{\nabla}_{\left[\tilde{X}, X_{i}\right]} X_{j}=\Theta\left(X_{i}\right) X_{j}+\Theta\left(X_{j}\right) X_{i} .
\end{aligned}
$$

By means of Lemma 2.1, Lemma 2.3, (12) and (15), the following relation is obtaind:

$$
\begin{aligned}
& -\left\{v^{b} H_{i b j}^{h}-X_{\bar{i}}\left(v^{\bar{b}}\right) G_{b j}^{h}-v^{\bar{b}} G_{i b j}^{h}-X_{\bar{i}} X_{j}\left(v^{\bar{h}}\right)\right. \\
& \left.\quad+\left(v^{b} R_{b j}^{m}-v^{\bar{b}} G_{b j}^{m}-X_{j}\left(v^{\bar{m}}\right)\right) C_{m i}^{h}\right\} X_{\bar{h}}=\delta_{j}^{h} \theta_{\bar{i}} X_{h}+\delta_{i}^{h} \theta_{j} X_{\bar{h}} .
\end{aligned}
$$


Thus, we obtain

$$
\theta_{\bar{i}}=0
$$

and

$$
\begin{aligned}
v^{b} H_{i b j}^{h} & -X_{\bar{i}}\left(v^{\bar{b}}\right) G_{b j}^{h}-v^{\bar{b}} G_{i b j}^{h}-X_{\bar{i}} X_{j}\left(v^{\bar{h}}\right) \\
& +\left(v^{b} R_{b j}^{m}-v^{\bar{b}} G_{b j}^{m}-X_{j}\left(v^{\bar{m}}\right)\right) C_{m i}^{h}=-\delta_{i}^{h} \theta_{j} .
\end{aligned}
$$

By means of Lemma 2.1, Lemma 2.3, (12), (16) and (18), the following can be presented:

$$
\begin{aligned}
\tilde{X}\left(C^{h}{ }_{i j}\right) & +C^{a}{ }_{i j}\left(v^{b} G_{b a}^{h}-X_{\bar{a}}\left(v^{\bar{h}}\right)\right)-\left(v^{b} G_{i b j}^{h}-X_{\bar{i}} X_{\bar{j}}\left(v^{\bar{h}}\right)\right) \\
& -C^{h}{ }_{i a}\left(v^{b} G^{a}{ }_{b j}-X_{\bar{j}}\left(v^{\bar{a}}\right)\right)-C^{h}{ }_{a j}\left(v^{b} G^{a}{ }_{b i}-X_{\bar{i}}\left(v^{\bar{a}}\right)\right)=0 .
\end{aligned}
$$

By means of Lemma2.1, Lemma2.3, (12) and (17) we have

$$
\begin{aligned}
& \tilde{X}\left(F_{j i}^{h}-P_{j i}^{h}\right)-\partial_{a} v^{h}\left(F_{j i}^{a}-P_{j i}^{a}\right)+\partial_{i} \partial_{j} v^{h} \\
& \quad+\partial_{j} v^{a}\left(F_{a i}^{h}-P_{a i}^{h}\right)+\partial_{i} v^{a}\left(F_{a j}^{h}-P_{a j}^{h}\right)=\delta_{i}^{h} \theta_{j}+\delta_{j}^{h} \theta_{i},
\end{aligned}
$$

and

$$
\begin{gathered}
\left(F_{j i}^{a}-P_{j i}^{a}\right)\left[v^{b} R_{b a}^{h}-v^{\bar{b}} G_{b a}^{h}-X_{a}\left(v^{\bar{h}}\right)\right]+X\left(g^{h t} R_{i t j}\right) \\
+g^{a t} R_{i t j}\left[v^{b} G_{b a}^{h}-X_{\bar{a}}\left(v^{\bar{h}}\right)\right]+\partial_{j} v^{a} g^{h t} R_{i t a}-X_{i}\left[v^{b} R_{b j}^{h}-v^{\bar{b}} G_{b j}^{h}-X_{j}\left(v^{\bar{h}}\right)\right] \\
-\left[v^{b} R_{b j}^{a}-v^{\bar{b}} G_{b j}^{a}-X_{j}\left(v^{\bar{a}}\right)\right] G_{a i}^{h}+\partial_{i} v^{b} g^{h t} R_{b t j}=0 .
\end{gathered}
$$

From (21), it can be concluded that

$$
£_{V} G^{h}=\theta_{i} y^{i} y^{h},
$$

i.e. $V=v^{h} \partial_{h}$ is an infinitesimal projective transformation on $(M, g)$. This completes the proof.

Proof of Theorem 1.1. Infinitesimal complete lift transformations is a subclass of infinitesimal fiber-preserving transformations. So, the similar method as in the proof of Proposition 4.1 is used. By means of Lemma 2.1, Lemma 2.4, relations (12), (15) and (18) the following can be given

$$
£_{V} G^{h}{ }_{j i}+2\left(£_{V} G_{j}^{t}\right) C_{i t}^{h}=\delta_{i}^{h} \theta_{j} .
$$

Contracting by $y^{i}$

$$
£_{V} G^{h}{ }_{j}=y^{h} \theta_{j},
$$

thus

$$
\left(£_{V} G_{j}^{t}\right) C_{i t}^{h}=0
$$

and, we obtain

$$
£_{V} G^{h}{ }_{j i}=\delta_{i}^{h} \theta_{j} .
$$

By means of Lemma 2.1 and Lemma 2.4 with the relations (12), (16) and (18):

$$
£_{V} C^{h}{ }_{j i}=0 .
$$

By taking in to account Lemma 2.1 and Lemma 2.4 with the relations (12) and (17), we can obtain

$$
£_{V}\left(F_{j i}^{h}-P_{j i}^{h}\right)=\delta_{i}^{h} \theta_{j}+\delta_{j}^{h} \theta_{i},
$$


and

$$
\begin{aligned}
£_{V} G_{t}^{h}\left(P_{j i}^{t}-F_{j i}^{t}\right) & +\partial_{j} v^{a} g^{h t} R_{i t a}+\partial_{i} v^{b} g^{h t} R_{b t j} \\
& +X_{i}\left(£_{V} G_{j}^{h}\right)+G_{t i}^{h}\left(£_{V} G_{j}^{h}\right)=0 .
\end{aligned}
$$

Contracting (26) by $y^{i} y^{j}$ and using (24) leads to obtain

$$
£_{V} G^{h}=0,
$$

thus, $V=v^{i} \partial_{i}$ is an infinitesimal affine transformation on $(M, g)$ and

$$
£_{V} G_{j}^{h}=£_{V} G_{j i}^{h}=0 .
$$

Substituting (27) in (24)

$$
\delta_{i}^{h} \theta_{j}=0 .
$$

Thus, $\theta_{i}=0$, i.e. $V^{c}$ is an infinitesimal affine transformation on $(T M, \tilde{g})$. This completes the proof.

Proof of Theorem 1.3. If $V^{c}$ is an infinitesimal projective transformation then from Theorem 1.1, one can see that $V$ is an affine. Let $(M, g)$ be a Landsberg manifold then

$$
F_{j i}^{h}=G_{j i}^{h} .
$$

If $V$ is an affine, then by use of (24), (26) and (28), it can be seen that $V^{c}$ is an infinitesimal affine transformation on $\left(T M_{0}, \tilde{g}\right)$. This completes the proof.

From Theorem 1.3, we have immediately the following remark.

Remark 4.1. Let $(M, g)$ be a Landsberg space, then, there is a one-to-one correspondence between complete lift projective vector fields on $\left(T M_{0}, \tilde{g}\right)$ and affine vector fields on $(M, g)$.

\section{REFERENCES}

[1] Akbar-Zadeh, H., "Champ de vecteurs projectifs sur le fibré unitaire", J. Math. pure et appl. 65 (1986), 47-79.

[2] Akbar-Zadeh, H., "Sur les espaces de Finsler à courbures sectionells constantes", Acad. Royale Belgique. Bull. de Sci. 5ème Sèrie LXXIV (1988), 281-322.

[3] Bejancu, A. and Farran, H. R., Geometry of pseudo-Finsler submanifolds, Mathematics and Its Applicaltions, Kluwer Academic Publishers, 2000.

[4] Bidabad, B., "Conformal vector fields on tangent bundle of Finsler manifolds", Balkan J. Geom. Appl. 111 (2006), 28-35.

[5] Gezer, A., "On infinitesimal conformal transformations of the tangent bundles with the synectic lift of Riemannian metric", Proc. Indian Acad. Sci. (Math. Sci.) Vol. 119 No. 3 (2009), 345-350.

[6] Gezer, A., "On infinitesimal holomorphically projective transformations on the tangent bundles with respect to the sasaki metric", proc. Estonian Acad. Sci. Phy. Math. 603 (2011), 149-157.

[7] Hasegawa, I. and Yamauchi, K., "Infinitesimal conformal transformations on tangent bundles with the lift metric I+II", Scientiae Mathematicae Japonicae 57 No. 1 (2003), 129-137; e7 437-445. 
[8] Hedayatian, S. and Bidabad, B., "Conformal vector fields on tangent bundle of a Riemannain manifold", Irannian Journal of Science and Technology, Trans. A Vol. 29 No. A3 (2005).

[9] Peyghan, E. and Heydari, A., "Conformal vector fields on tangent bundle of a Riemannain manifold", J. Math. Anal. Appl. 347 (2008), 136-142.

[10] Peyghan, E. Razavi, A. and Heydari, A., "Conformal vector fields on tangent bundle with a special lift Finsler metric", Irannian Journal of Science and Technology, Trans. A Vol. 32 No. A1 (2008).

[11] Shen, Z., Differential Geometry of Spray and Finsler Spaces, Kluwer Academic Pubblishers, 2001.

[12] Yamauchi, K., "On Riemannian manifolds admitting infinitesimal projective tansformations", Hokkaido Math. J. Vol. 16 (1987), 115-125.

[13] Yamauchi, K., "On infinitesimal conformal transformations of the tangent bundles over Riemannian manifolds", Ann. Rep. Asahikawa. Med. Coll. 16 (1995), 1-6.

[14] Yamauchi, K., "On infinitesimal projective transformations of the tangent bundles with the complete lift metric over Riemannian manifolds", Ann. Rep. Asahikawa. Med. Coll. Vol. 19 (1998), 49-55.

[15] Yamauchi, K., "On conformal transformation in tangent bundles", Hokkaido Math. J. Vol. 30 (2001), 359-372.

[16] Yano, K., The Theory of Lie Derivatives and Its Applications, Bibliotheca mathematica, North Holland Pub. Co., 1957.

[17] Yano, K. and Ishihara, S., Tangent and Cotangent Bundles, New York: Marcel Dekker, Inc., 1973. 